\title{
A Multi-Institutional Analysis of Elderly Patients Undergoing a Liver Resection for Intrahepatic Cholangiocarcinoma
}

\author{
ALESSANDRO VITALE, MD, PhD, ${ }^{1}$ GAYA SPOLVERATO, $\mathrm{MD}^{2}{ }^{2}$ FABIO BAGANTE, $\mathrm{MD}^{2}{ }^{2}$ FAIZ GANI, $\mathrm{MD}^{2}{ }^{2}$ \\ IRINEL POPESCU, MD, ${ }^{3}$ HUGO P. MARQUES, $\mathrm{MD}^{4}{ }^{4}$ LUCA ALDRIGHETTI, $\mathrm{MD}^{5}{ }^{5}$ T. CLARK GAMBLIN, $\mathrm{MD}^{6}$ \\ SHISHIR K. MAITHEL, MD, ${ }^{7}$ CHARBEL SANDROUSSI, $\mathrm{MD}_{,}{ }^{8}$ TODD W. BAUER, MD, ${ }^{9}$ FENG SHEN, $\mathrm{MD}^{10}$ \\ GEORGE A. POULTSIDES, MD, ${ }^{11}$ J. WALLIS MARSH, MD, ${ }^{12}$ AND TIMOTHY M. PAWLIK, MD, MPH, $\mathrm{PhD}^{2} *$ \\ ${ }^{1}$ Unità di Chirurgia Epatobiliare e Trapianto Epatico, Azienda Ospedaliera-Università di Padova, Padova, Italy \\ ${ }^{2} J o h n s$ Hopkins University School of Medicine, Baltimore, Maryland \\ ${ }^{3}$ Fundeni Clinical Institute, Bucharest, Romania \\ ${ }^{4}$ Curry Cabral Hospital, Lisbon, Portugal \\ ${ }^{5}$ Ospedale San Raffaele, Milan, Italy \\ ${ }^{6}$ Medical College of Wisconsin, Milwaukee, Wisconsin \\ 'Emory University, Atlanta, Georgia \\ ${ }^{8}$ University of Sydney, Sydney, Australia \\ ${ }^{9}$ University of Virginia, Charlottesville, Virginia \\ ${ }^{10}$ Eastern Hepatobiliary Surgery Hospital, Shanghai, China \\ ${ }^{11}$ Stanford University, Stanford, California \\ ${ }^{12}$ University of Pittsburgh Medical Center, Pittsburgh, Pennsylvania
}

Background: Little is known regarding postoperative outcomes of elderly patients undergoing liver surgery for intrahepatic cholangiocarcinoma (ICC).

Methods: Five hundred and eighty-four patients undergoing liver resection for ICC between 1990 and 2015 were identified. Perioperative morbidity, mortality, overall survival (OS), and disease-free survival (DFS) were compared between elderly $(>70$ year, $n=129)$ and non-elderly $(\leq 70$ years, $\mathrm{n}=455)$ patients.

Results: Older patients had a higher incidence of complications (elderly vs. non-elderly; $52.7 \%$ vs. $42.6 \%$; $P=0.03$ ), as well as major complications (elderly vs. non-elderly; $24.0 \%$ vs. $14.9 \% ; P=0.01)$; 30 -day $(0.1 \%$ vs. $3.3 \% ; P>0.05)$, and 90 -day mortality $(2.3 \%$ vs. $5.5 \%$; $P>0.05)$ were comparable. Five-year OS and DFS were comparable between the elderly and non-elderly patients (OS, $13.3 \%$ vs. 24.4\%; and DFS; $7.3 \%$ vs. $12.0 \% ; P>0.05)$. On propensity score matching, DFS and OS were also comparable among non-elderly versus elderly patients. Poor tumor grade was associated with worse DFS among elderly patients $(\mathrm{HR}=1.6,95 \% \mathrm{CI} 1.0-2.6 ; P=0.04)$, whereas periductal invasion $(\mathrm{HR}=1.9$, 95\% CI 1.1-3.5; $P=0.03)$ and nodal disease (HR $=2.3,95 \%$ CI 1.3-3.9; $P=0.003)$ were predictive of shorter DFS among non-elderly patients. Conclusion: Elderly patients undergoing liver surgery for ICC demonstrated an increased risk of perioperative complications, but comparable long-term DFS and OS compared with younger patients. Rather, tumor characteristics were more predictive of worse long-term outcomes. J. Surg. Oncol. 2016;113:420-426. (C) 2016 Wiley Periodicals, Inc.

KEY WoRDS: intrahepatic cholangiocarcinoma; surgery; hepatectomy; liver resection; elderly

\section{INTRODUCTION}

Cholangiocarcinoma accounts for $3 \%$ of all gastrointestinal malignancies and represents the second most common primary liver tumor [1-6]. In particular, the incidence of intrahepatic cholangiocarcinoma (ICC) has been steadily increasing with the incidence in the United States varying from 0.72 to 0.88 per 100,000 $[1-4,7]$. Of note, the incidence of ICC increases with age, as older patients demonstrate an incidence that can be almost twofold higher than younger patients $[1-4,7]$. Despite recent advances in care, surgery remains the preferred therapeutic option for patients with ICC, conferring a 5-year survival between $30 \%$ and $35 \%$ [8]. Several clinicopathological factors have been associated with poor postoperative outcomes among patients undergoing surgery for ICC including tumor size, presence of multiple tumors, cirrhosis, lymph node metastasis, and macrovascular invasion [8-11]. Several other reports have suggested that patient age may also be a potential negative prognostic factor [9-13].

Older patients often have a higher incidence of medical comorbidities, worse performance status, as well as decreased functional reserve that may place them at a particular risk for worse postoperative outcomes [1]. However, while several studies have suggested an increased incidence of postoperative complications among elderly patients undergoing major liver surgery [14-16], other studies have failed to note an increased risk of liver resection among the elderly [17-20]. In addition, the impact of elder age on postoperative outcomes and long-term survival among elderly patients undergoing liver surgery for ICC, in particular, remains poorly defined. Given this,

A.Vitale and G. Spolverato contributed equally to this manuscript.

*Correspondence to: Timothy M. Pawlik, MD, MPH, PhD, FACS, Division of Surgical Oncology, John L. Cameron, Department of Surgery Johns Hopkins Hospital 600 N. Wolfe Street Blalock 688 Baltimore, MD 21287. Fax: (410) 502-2388. E-mail: tpawlik1@jhmi.edu

Received 1 September 2015; Accepted 13 December 2015 DOI 10.1002/jso.24148

Published online 12 January 2016 in Wiley Online Library (wileyonlinelibrary.com). 
we sought to evaluate the effect of age on postoperative outcomes among patients undergoing liver surgery for ICC. In particular, we aimed to define perioperative outcomes including postoperative complication and 90-day mortality, as well as disease-free survival (DFS) and overall survival (OS) among elderly patients ( $>70$ years) undergoing liver surgery for ICC using a large multi-center international database.

\section{METHODS}

\section{Study Population and Data Collection}

Patients undergoing a hepatic resection for histologically confirmed ICC between 1990 and 2013 were identified using a multi-institutional database of 671 patients collected at 12 major hepato-biliary centers in North America, Europe, Australia and, Asia (Johns Hopkins Hospital, Baltimore, Maryland; Medical College of Wisconsin, Milwaukee, Wisconsin; Stanford University, Stanford, California; University of Virginia, Charlottesville, Virginia; Emory University, Atlanta, Georgia; University of Pittsburgh, Pittsburgh, Pennsylvania; Fundeni Clinical Institute of Digestive Disease, Bucharest, Romania; Curry Cabral Hospital, Lisbon, Portugal; Hopitaux Universitaires De Geneve, Geneve, Switzerland; Ospedale San Raffaele, Milan, Italy; Royal Prince Alfred Hospital, University of Sydney, Sydney, Australia; and Eastern Hepatobiliary Surgery Hospital, Shanghai, China). Patients who underwent a palliative procedure $(n=39)$ as well as patients who were diagnosed with metastatic disease $(n=48)$ were excluded from the analysis. Thus, the final study group included 584 ICC patients undergoing resection with curative intent. The Institutional Review Board of each participating institution approved the study.

Demographic and clinicopathological data collected for each patient included age, gender, body mass index (BMI), American Society of Anesthesiologist (ASA), physical classification score, preexisting comorbidity and, the presence of preoperative cirrhosis or jaundice. Tumor-specific characteristics such as tumor size, number of lesions, tumor grade, lymph node involvement, macroscopic or microscopic vascular invasion, perineural invasion, as well as direct invasion of contiguous organs were collected for each patient. Tumor stage was classified according to the 7th edition of the American Joint Committee on Cancer (AJCC) staging system [21]. Operative details collected for each patient included the type and extent of hepatic resection, receipt of lymphadenectomy, margin status, operative time, estimated blood loss (EBL), and receipt of intraoperative blood transfusion. Major hepatectomy was defined as the resection of three or more liver segments according to Couinaud's classification [22]. Resection margin and nodal status were described according to the final histopathology report.

The primary outcomes of interest were DFS and OS, both of which were calculated from the date of surgery. Secondary, postoperative outcomes included the development of postoperative complications, length-of-stay (LOS) for the admission in which the surgery was performed, postoperative mortality within 30 and 90 days following surgery, 30-day readmission, the receipt of adjuvant chemotherapy or radiotherapy, and recurrence of disease. Postoperative complications were classified according to the Clavien-Dindo classification system whereby grades 1 and 2 complications were categorized as "minor complications" and grades 3 and 4 complications classified as "major complications" [23]. Recurrence was defined as the presence of a biopsy-proven tumor or an image that was highly suspicious of tumor recurrence.

\section{Statistical Analysis}

Discrete variables were described as medians with interquartile range (IQR) and compared using the Mann-Whitney $U$ test. Categorical variables were described as totals and frequencies and compared using the $\chi^{2}$ test or Fisher's exact test as appropriate. The nearest neighbor-matching algorithm was used to calculate a propensity score to create a comparable, matched cohort of patients of elderly ( $>70$ years), and non-elderly patients ( $\leq 70$ years). The propensity score was calculated using a multivariable logistic regression analysis with the patient age group ( $>70$ years) as the dependent variable. The independent variables were factors that were statistically different in the multivariable survival analysis comparing the two groups $(>70$ years vs. $\leq 70$ years).

DFS and OS time were calculated using the Kaplan-Meier method and compared between the two patient groups using the log-rank test. Univariable and multivariable logistic regression analyses were performed to assess the association of patient and disease characteristics with DFS and OS within each patient age group. Variables found to be statistically significant on univariable analysis were included in the multivariable model to assess for potential associations and confounding. Statistical analyses were performed using JMP software version 9.0.1 (1989-2010 SAS Institute Inc.) and STATA version 13.1 (StataCorp LP, College Station, TX). All tests were two-tailed and statistical significance was defined as $P<0.05$.

\section{RESULTS}

\section{Demographic and Clinicopathological Characteristics}

A total of 584 patients underwent hepatic resection for ICC between 1990 and 2013 and met inclusion criteria. The median age of the cohort was 60 years (IQR: $50.8-69.0)$ and $53.3 \%(\mathrm{n}=311)$ were male. Over a fifth of patients were $>70$ years $(n=129,22.1 \%)$ whereas $77.9 \%$ of patients were $\leq 70$ years $(n=455,77.9 \%)$ at the time of diagnosis. Significant differences in baseline characteristics were noted between elderly patients ( $>70$ years) and younger patients ( $\leq 70$ years). Patients $>70$ years old who underwent surgery were proportionally less likely to present with a bi-lobar tumor (non-elderly vs. elderly; $34.3 \%$ vs. $25.6 \%$, $P=0.038$ ). Similarly, major vascular invasion was also more common among younger patients (non-elderly vs. elderly; $14.9 \%$ vs. $7.8 \%$, $P=0.039$, Table I).

\section{Postoperative Complications, LOS, 30- and 90-Day Mortality, and Adjuvant Therapies}

Among the unmatched cohort of patients, LOS was comparable between non-elderly and elderly patients (median LOS: non-elderly vs. elderly; 10 days [IQR: $6-15$ ] vs. 8 days [IQR: $6-14$ ], $P=0.115$ ). Of note, elderly patients were more likely to develop a postoperative complication compared with non-elderly patients (non-elderly vs. elderly; $42.6 \%$ vs. $52.7 \%, P=0.027$ ). Additionally, elderly patients were also proportionally more likely to develop a major complication defined as Clavien-Dindo grade $\geq 2$ (non-elderly vs. elderly; $14.9 \%$ vs. $24.0 \%, P=0.0013$ ), as well as be readmitted within 30-day of discharge (non-elderly vs. elderly; $11.2 \%$ vs. $16.3 \%, P=0.046$ ). Postoperative mortality within 30 - and 90-days was similar between the two groups (both $P>0.05$ ). Compared with older patients, younger patients were more likely to receive adjuvant chemotherapy (young vs. elderly; $55.4 \%$ vs. $37.2 \%, P<0.001$, Table IIa).

To minimize potential confounding due to case mix, a propensityscore matched cohort of comparable patients was created including 129 patients aged $\leq 70$ years and 129 patients $>70$ years. Of note, among the cohort of matched patients, LOS, 30-day readmission, and postoperative mortality were similar between the two patient groups (all $P>0.05$, Table IIb). On matched analysis, elderly patients were proportionally more likely to develop a major postoperative complication compared with non-elderly patients (non-elderly vs. elderly; $14.0 \%$ vs. $24.0 \%, P=0.028$ ). In contrast, elderly patients were 
TABLE I. Clinical and Pathologic Features of Unmatched Patients $(n=584)$

\begin{tabular}{|c|c|c|c|}
\hline Characteristic & Age $\leq 70$ years $(\mathrm{n}=455)$ & Age $>70$ years $(n=129)$ & $P$-value \\
\hline Age, year, median (IQR) & $55.9(48.6-63.0)$ & $74.0(71.2-77.3)$ & $<0.0001$ \\
\hline Female & $211(46.4 \%)$ & $62(48.1 \%)$ & 0.7647 \\
\hline Non-Caucasian race & $88(19.3 \%)$ & $8(6.2 \%)$ & 0.0002 \\
\hline Cirrhosis & $42(9.2 \%)$ & $16(12.4 \%)$ & 0.3167 \\
\hline ASA class $>2$ & $290(63.7 \%)$ & $86(66.7 \%)$ & 0.6028 \\
\hline Tumor size, $\mathrm{cm}$, median (IQR) & $7.0(5.0-9.4)$ & $6.0(4.5-9.0)$ & 0.1051 \\
\hline Multiple lesions & $143(31.4 \%)$ & $43(33.3 \%)$ & 0.6703 \\
\hline Bi-lobar tumor & $156(34.3 \%)$ & $33(25.6 \%)$ & 0.0379 \\
\hline CA 19-9, units/ml, median (IQR) & $145.4(23.6-784.0)$ & $93.4(7.5-514.9)$ & 0.3840 \\
\hline Vascular invasion (major or minor) & $155(34.1 \%)$ & $39(30.2 \%)$ & 0.4591 \\
\hline Major vascular invasion & $68(14.9 \%)$ & $10(7.8 \%)$ & 0.0393 \\
\hline Minor vascular invasion & $132(29.0 \%)$ & $37(28.7 \%)$ & 1.000 \\
\hline Biliary invasion & $68(14.9 \%)$ & $16(12.4 \%)$ & 0.5698 \\
\hline Poor tumor grade & $121(26.6 \%)$ & $31(24.0 \%)$ & 0.6494 \\
\hline Perineural invasion & $87(19.1 \%)$ & $32(24.8 \%)$ & 0.0997 \\
\hline Direct invasion of contiguous organs & $52(11.4 \%)$ & $10(7.8 \%)$ & 0.1498 \\
\hline Periductal invasion & $60(13.2 \%)$ & $12(9.3 \%)$ & 0.1503 \\
\hline Nodal status & & & 0.4496 \\
\hline No & $132(29.0 \%)$ & $38(29.5 \%)$ & \\
\hline N1 & $93(20.4 \%)$ & $25(19.4 \%)$ & \\
\hline $\mathrm{Nx}$ & $230(50.6 \%)$ & $66(51.2 \%)$ & \\
\hline AJCC $\mathrm{T}$ stage & & & 0.5497 \\
\hline $\mathrm{T} 1$ & $187(41.1 \%)$ & $54(41.9 \%)$ & \\
\hline $\mathrm{T} 2 \mathrm{a}$ & $71(15.6 \%)$ & $21(16.3 \%)$ & \\
\hline $\mathrm{T} 2 \mathrm{~b}$ & $105(23.1 \%)$ & $34(26.4 \%)$ & \\
\hline $\mathrm{T} 3$ & $32(7.0 \%)$ & $8(6.2 \%)$ & \\
\hline T4 & $60(13.2 \%)$ & $12(9.3 \%)$ & \\
\hline $\mathrm{AJCC}^{\mathrm{a}}$ & & & 0.7672 \\
\hline I & $218(37.4 \%)$ & $45(34.9 \%)$ & \\
\hline II & $179(30.6 \%)$ & $45(34.9 \%)$ & \\
\hline III & $25(4.3 \%)$ & $6(4.7 \%)$ & \\
\hline $\mathrm{IVa}^{\mathrm{b}}$ & $162(27.7 \%)$ & $33(25.6 \%)$ & \\
\hline $\mathrm{R} 1$ margin & $83(18.2 \%)$ & $27(20.9 \%)$ & 0.2838 \\
\hline Major surgical resection ( $\geq 3$ segments) & $317(69.7 \%)$ & $91(70.5 \%)$ & 0.4706 \\
\hline Intra-operative blood transfusion & $170(37.4 \%)$ & $44(34.1 \%)$ & 0.2844 \\
\hline Intra-operative blood loss, ml, median (IQR) & $500(250-900)$ & $550(400-1075)$ & 0.1042 \\
\hline Operative time, min, median (IQR) & $230(180-315)$ & $279(180-360)$ & 0.1021 \\
\hline
\end{tabular}

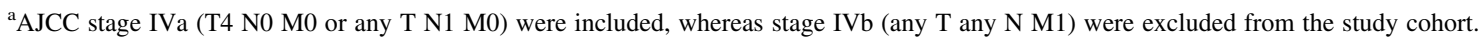

${ }^{\mathrm{b}}$ Includes tumors with periductal-infiltrating or mixed mass-forming and periductal-infiltrating growth pattern.

less likely to receive both adjuvant chemo- and radiation therapy (chemotherapy: non-elderly vs. elderly; $49.6 \%$ vs. $37.2 \%, P=0.028$, radiation therapy: non-elderly vs. elderly; $23.3 \%$ vs. $6.9 \%, P<0.001$, Table IIb).

\section{Disease Free and Overall Survival}

The median follow-up for the unmatched cohort was 50.6 months (IQR: 31.2-91.9) while the median follow-up was 43.4 months (IQR:

TABLE II. (a) Postoperative Outcomes in Unmatched Cohort; (b) Postoperative Outcomes in Matched Cohort

\begin{tabular}{|c|c|c|c|}
\hline \multicolumn{4}{|l|}{$\mathrm{a}$} \\
\hline Variables & Age $\leq 70$ years $(n=455)$ & Age $>70$ years $(n=129)$ & $P$-value \\
\hline Length of stay, days, median (IQR) & $10(6.0-15.0)$ & $8(6.0-14.0)$ & 0.1145 \\
\hline Any complication & $194(42.6 \%)$ & $68(52.7 \%)$ & 0.0270 \\
\hline Clavien score $>2$ & $68(14.9 \%)$ & $31(24.0 \%)$ & 0.0126 \\
\hline Readmission & $51(11.2 \%)$ & $21(16.3 \%)$ & 0.0455 \\
\hline Adjuvant chemotherapy & $252(55.4 \%)$ & $48(37.2 \%)$ & 0.0002 \\
\hline Adjuvant radiotherapy & $57(12.5 \%)$ & $9(6.9 \%)$ & 0.0500 \\
\hline 30-day mortality & $15(3.3 \%)$ & $1(0.1 \%)$ & 0.0988 \\
\hline 90-day mortality & $25(5.5 \%)$ & $3(2.3 \%)$ & 0.0994 \\
\hline \multicolumn{4}{|l|}{ b } \\
\hline Variables & Age $\leq 70$ years $(n=129)$ & Age $>70$ years $(n=129)$ & $P$-value \\
\hline Length of stay, days, median (IQR) & $9(6.0-15.5)$ & $8(6.0-14.0)$ & 0.3096 \\
\hline Any complication & $54(41.9 \%)$ & $68(52.7 \%)$ & 0.0524 \\
\hline Clavien score $>2$ & $18(14.0 \%)$ & $31(24.0 \%)$ & 0.0280 \\
\hline Readmission & $15(11.5 \%)$ & $21(16.3 \%)$ & 0.0567 \\
\hline Adjuvant chemotherapy & $64(49.6 \%)$ & $48(37.2 \%)$ & 0.0297 \\
\hline Adjuvant radiotherapy & $30(23.3 \%)$ & $9(6.9 \%)$ & 0.0002 \\
\hline 30-day mortality & $6(4.7 \%)$ & $1(0.1 \%)$ & 0.0599 \\
\hline 90-day mortality & $7(5.4 \%)$ & $3(2.3 \%)$ & 0.1670 \\
\hline
\end{tabular}



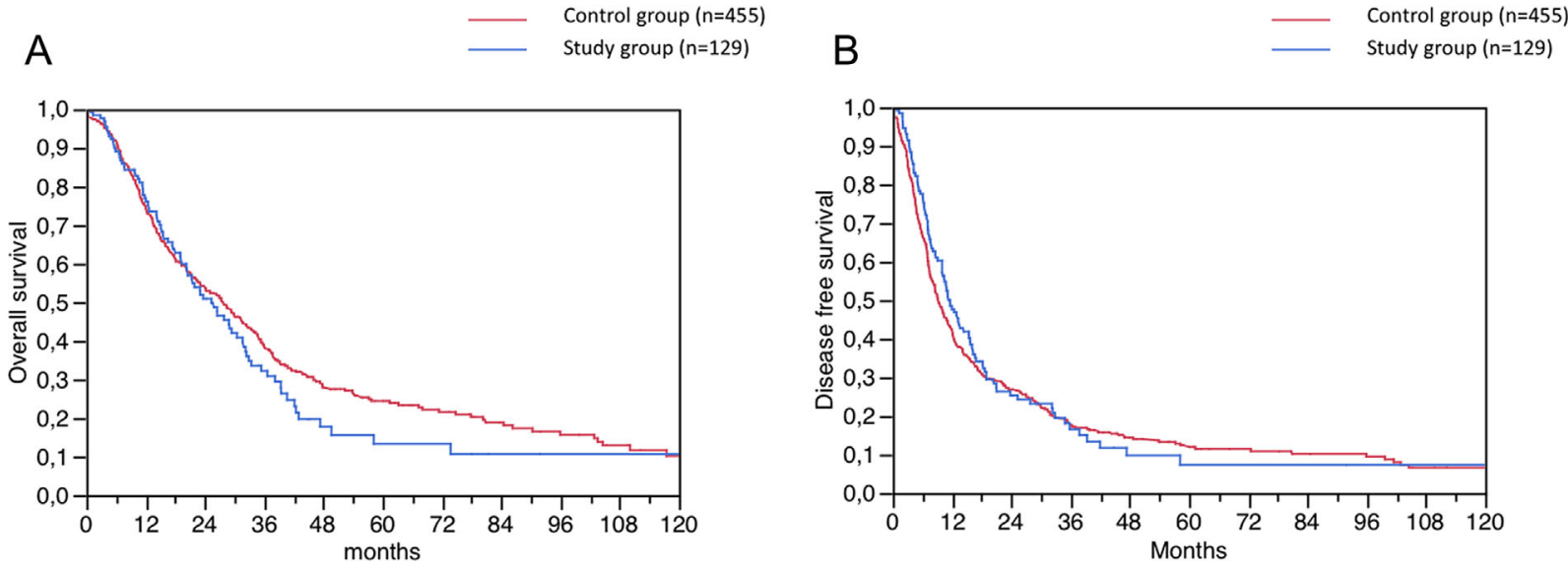

Fig. 1. Kaplan-Meier curves comparing: (a) overall survival and (b) disease-free survival among elderly versus non-elderly in the unmatched cohort.

25.6-81.4) for the propensity-matched cohort. For the entire unmatched cohort, the 1-, 3-, and 5-year DFS was $43.6 \%, 17.6 \%$ and $11.3 \%$, respectively, and the 1-, 3-, and 5-year OS was $75.4 \%, 37.4 \%$, and $22.5 \%$, respectively. On unadjusted analyses, there were no differences in DFS or OS noted between non-elderly and elderly patients: the median DFS among elderly patients was 11.6 months (IQR: 6.4-25.3) versus 9.2 months (IQR: 4.6-27.0) among non-elderly patients $(P=0.470$, Fig. 1$)$; the median OS was 25.3 months $(12.6-40.6)$ versus 27.5 months (IQR: $11.8-57.3)$, respectively $(P=0.286$, Fig. 1). On propensity score matching, DFS and OS were also comparable among non-elderly versus elderly patients. Specifically, the median DFS among non-elderly patients was 9.1 months (IQR: 5.2-26.0; $P=0.42$, Fig. 2), while median OS in the same subgroup was 27.3 months (IQR: $12.1-63.2 ; P=-0.357$, Fig. 2).

Multivariable logistic regression analysis was performed to assess patient and disease factors predictive of OS and DFS among non-elderly and elderly patients. Of note, tumor grade (HR 1.7, 95\% CI 1.0-2.9; $P=0.04$ ) and periductal invasion (HR $2.5,95 \%$ CI $1.1-5.3 ; P=0.03$ ) were predictive of a poor OS among elderly patients, while CA$19.9>125 \mathrm{U} / \mathrm{ml}(\mathrm{HR} 1.8,95 \% \mathrm{CI} 1.1-2.9 ; P=0.02)$, vascular invasion (HR 2.3, 95\% CI 1.3-4.1; $P=0.007$ ), poor tumor grade (HR 1.9, 95\% CI $1.1-3.5 ; P=0.03$ ), periductal invasion (HR 4.2, 95\% CI 1.9-9.1; $P<0.001$ ), and receipt of adjuvant radiotherapy (HR 2.0, 95\% CI 1.1-
$3.4 ; P=0.01)$ were more important predictors of worse OS among nonelderly patients (Table III). Poor tumor grade was the only clinicopathological characteristic associated with a worse DFS among elderly patients (HR 1.6, 95\% CI 1.0-2.6; $P=0.04$ ), while periductal invasion (HR 1.9, 95\% CI $1.1-3.5 ; P=0.03$ ) as well as nodal disease (HR 2.3, 95\% CI 1.3-3.9; $P=0.003$ ) were predictive of a shorter DFS among non-elderly patients (Table III). To further assess the relationship between age, nodal disease and DFS, patients were stratified by node status and DFS was stratified by non-elderly versus elderly. Interestingly, among patients with nodal metastasis, DFS was better among elderly patients when compared with DFS among non-elderly patients $(P=0.04$, Fig. $3 \mathrm{~A})$; in contrast, DFS was similar among non-elderly and elderly patients who had no lymph node metastasis (Fig. 3B)

\section{DISCUSSION}

There has been a marked increase in the incidence of ICC, making it the second most common primary liver cancer [1-6]. As surgical management is the gold standard of care for patients presenting with ICC, and given that the population is aging, it is expected that the number of patients undergoing a hepatic resection for ICC will increase [1-6]. Although several studies have examined hepatic resection among
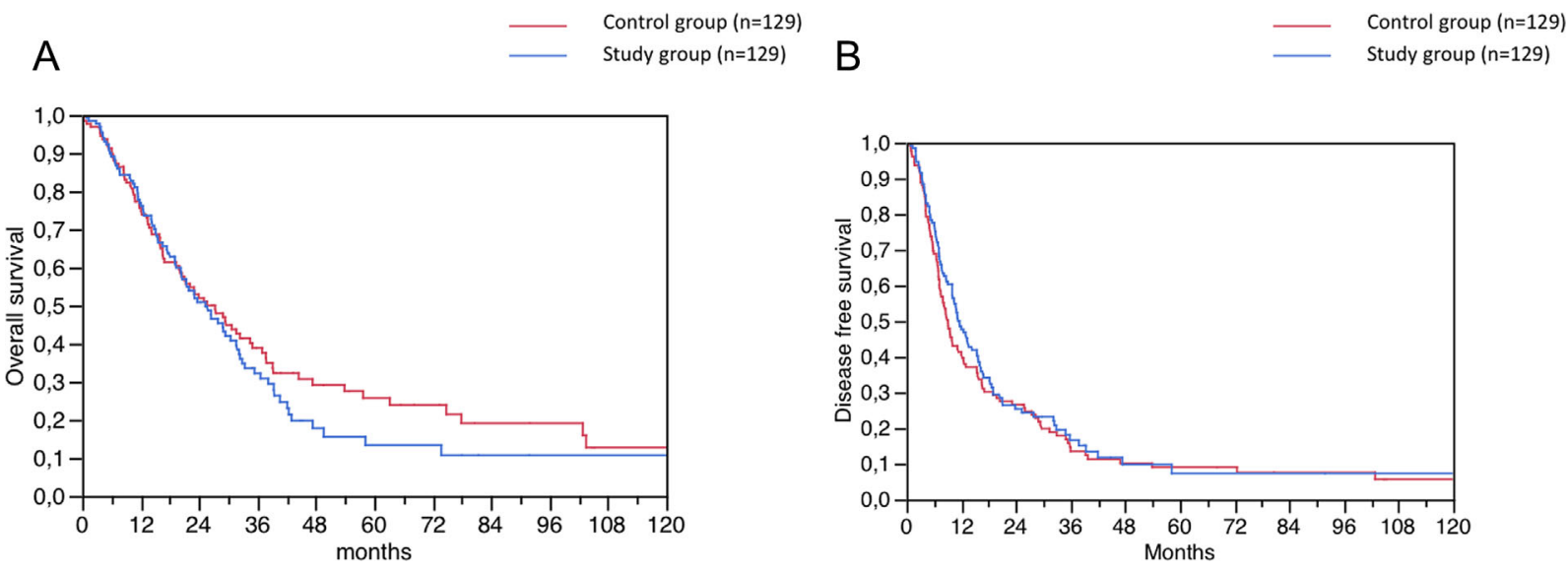

Fig. 2. Kaplan-Meier curves comparing: (a) overall survival and (b) fisease-free survival among elderly versus non-elderly in the propensityscore matched cohort. 
TABLE III. Multivariate Overall and Disease Free Survival Analyses in Study and Control Groups After Propensity Score Analysis

\begin{tabular}{|c|c|c|}
\hline Variables & Age $\leq 70$ years HR $(95 \% \mathrm{CI}), P$-value & Age $>70$ years $\mathrm{HR}(95 \% \mathrm{CI}), P$-value \\
\hline \multicolumn{3}{|l|}{ Overall Survival } \\
\hline Non Caucasian race & $0.85(0.37-2.21), 0.7201$ & $0.33(0.16-0.82), 0.0194$ \\
\hline Size $>5 \mathrm{~cm}$ & $1.58(0.91-2.81), 0.1063$ & $1.48(0.86-2.57), 0.1554$ \\
\hline CA $19-9>125 \mathrm{U} / \mathrm{ml}$ & 1.80 (1.11-2.92), 0.0162 & $1.16(0.72-1.86), 0.5465$ \\
\hline Vascular invasion (major or minor) & $2.28(1.26--4.11), 0.0068$ & $1.06(0.58-1.89), 0.8362$ \\
\hline Biliary invasion & $0.55(0.24-1.15), 0.1127$ & $0.53(0.24-1.11), 0.0913$ \\
\hline Poor tumor grade & $1.95(1.08-3.47), 0.0279$ & $1.75(1.01-2.95), 0.0457$ \\
\hline Direct invasion of contiguous organs & $1.50(0.51-3.94), 0.4435$ & $0.99(0.39-2.14), 0.9722$ \\
\hline Periductal invasion $^{\mathrm{a}}$ & $4.22(1.90-9.06), 0.0005$ & $2.46(1.07-5.30), 0.0345$ \\
\hline Positive nodal status & $1.59(0.88-2.77), 0.1235$ & $1.25(0.68-2.20), 0.4645$ \\
\hline Major surgical resection & $1.01(0.55-1.90), 0.9796$ & $1.03(0.60-1.80), 0.9167$ \\
\hline Adjuvant radiotherapy & $2.01(1.15-3.38), 0.0148$ & $2.18(0.88-4.67), 0.0886$ \\
\hline \multicolumn{3}{|l|}{ Disease Free Survival } \\
\hline Non Caucasian race & $0.43(0.20-1.03), 0.0590$ & $0.38(0.18-0.93), 0.0364$ \\
\hline Size $>5 \mathrm{~cm}$ & $1.22(0.77-1.96), 0.4004$ & $1.43(0.88-2.36), 0.1498$ \\
\hline Multiple lesions & $1.23(0.80-1.88), 0.3495$ & $1.00(0.64-1.52), 0.9874$ \\
\hline Vascular invasion (major or minor) & $1.44(0.86-2.38), 0.1662$ & 1.19 (0.71-1.99), 0.5049 \\
\hline Poor tumor grade & $1.87(1.12-3.05), 0.0170$ & $1.65(1.02-2.62), 0.0427$ \\
\hline Direct invasion of contiguous organs & $1.43(0.59-3.09), 0.4079$ & $1.08(0.49-2.12), 0.8273$ \\
\hline Periductal invasion ${ }^{\mathrm{a}}$ & $1.97(1.07-3.51), 0.0301$ & $1.65(0.80-3.19), 0.1690$ \\
\hline Positive nodal status & $2.31(1.34-3.87), 0.0031$ & $1.14(0.65-1.94), 0.6407$ \\
\hline Major surgical resection & $1.15(0.69-1.94), 0.6060$ & $1.25(0.78-2.06), 0.3598$ \\
\hline Adjuvant chemotherapy & $0.81(0.52-1.24), 0.3305$ & $1.52(0.98-2.36), 0.0640$ \\
\hline Adjuvant radiotherapy & $1.62(1.01-2.54), 0.0465$ & $1.10(0.45-2.28), 0.8188$ \\
\hline
\end{tabular}

${ }^{\mathrm{a}}$ Includes tumors with periductal-infiltrating or mixed mass-forming and periductal-infiltrating growth pattern.

elderly patients, the results were disparate and no previous study specifically examined elderly patients undergoing surgery for ICC. As such, we sought to use a large multinational cohort of 584 patients to assess postoperative outcomes among elderly patients undergoing hepatic resection for ICC. The current study is important because it noted that although elderly patients were more likely to develop severe postoperative complications, and were less likely to receive adjuvant therapy, no differences in DFS or OS were noted between elderly versus non-elderly patients. Rather than age, disease characteristics such as tumor invasion and nodal status were more predictive of long-term outcome among all patients.

Given the increasing aged surgical population, it is not surprisingly that the number of elderly patients undergoing hepatic resection for treatment of liver cancer is progressively increasing [24]. Although surgical resection remains the mainstay for potential cure for liver tumors, it is unclear whether the benefits of surgery are conferred to the elderly patient. Previous reports have identified elderly patients are a potential high-risk group following liver surgery due to greater preexisting comorbidity, poly-pharmacy, and decreased physiological reserve $[1,2]$. Consistent with these findings, the current study noted that, compared with non-elderly patients, elderly patients were more likely to develop a severe postoperative complication. Interestingly, this difference was also noted after patients were matched on several patient and disease characteristics. These data suggest that age may be a potential independent risk factor for developing postoperative complications. In a recent meta-analysis, Phan et al. reported that elderly patients were more likely to develop major postoperative complications including pneumonia, heart failure, surgical site infections, and renal failure compared with younger patients [25]. However, the majority of previous studies have only included data on postoperative outcomes among elderly patients undergoing liver surgery for hepatocellular carcinoma or colorectal liver metastasis. As such, to our knowledge, the current study represents the first study to assess postoperative outcomes among elderly patients undergoing liver surgery for the management of ICC.
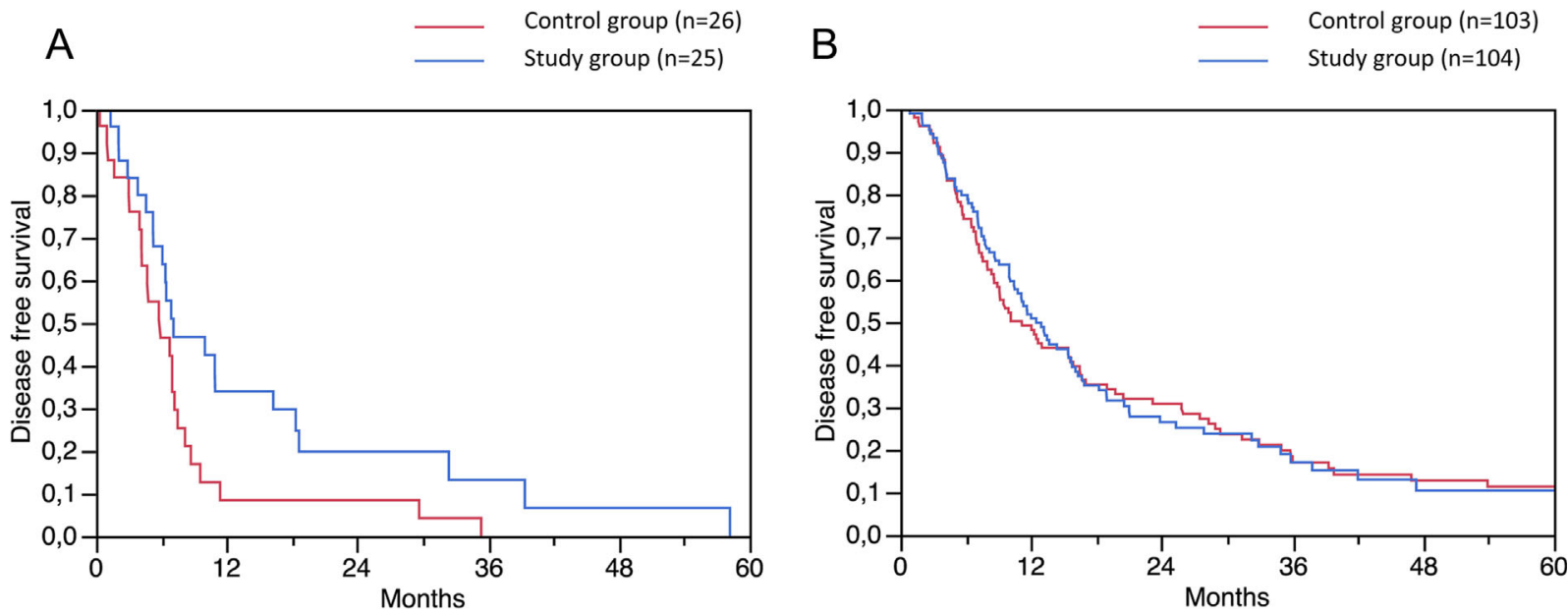

Fig. 3. Kaplan-Meier curves comparing disease-free survival among the elderly versus non-elderly in the propensity-score matched cohort among patients with (a) and without lymph node metastasis (b). 
Decisions to treat elderly patients with cancer are multifaceted and complex. Specifically, given the high postoperative morbidity associated with oncological surgery, elderly patients represent a unique challenge for healthcare providers. Similarly, several previous reports have demonstrated that treatment decisions can be largely driven by patient age with elderly patients being less likely to receive surgical or medical treatments compared with younger patients who have comparable disease [26-28]. For example, in a large survey of primary care providers, age at diagnosis was independently associated with the decision not to refer patients with advanced cancer for further treatment [26]. In a separate study, Guadagnoli et al. reported that up to $50 \%$ of elderly patients presenting with breast cancer were "undertreated" despite evidence suggesting that these patients would have benefited from treatment [29]. In the current study, using a matched cohort of elderly and non-elderly patients, we noted that elderly patients were less likely to receive adjuvant treatment in the form of chemotherapy or radiation therapy compared with non-elderly patients presenting with similar disease. Taken together, the findings of the current study as well as past reports, strongly suggest that patient age may influence a provider's choice to offer peri-operative adjuvant therapy to elderly patients. The reason for this is undoubtedly multi-factorial and may be related to the higher incidence of complications in the elderly or physician concern that therapy will not be as well tolerated in older patients. Although the role of adjuvant therapy for ICC is poorly defined, it is interesting that elderly patients were less likely to undergo adjuvant therapy, yet had similar DFS and OS in the matched cohort. Moreover, adjuvant chemotherapy had a negative prognostic impact in elderly patients and adjuvant radiotherapy decreased survival in nonelderly patients. These findings were undoubtedly influenced by selection bias (i.e., patients with more aggressive tumors received adjuvant therapy). In addition, these findings corroborate the modest effect that adjuvant therapy likely has on the oncological outcome of patients undergoing liver resection for ICC. In the future, more robust clinical tools will need to be employ to more accurately identify, which elderly patients would benefit the most from adjuvant therapies.

Another interesting finding of the current study was that both OS and DFS were comparable among elderly and non-elderly patients. In contrast, tumor characteristics were more predictive of worse survival. Interestingly, there were several factors that seemed to have a differential prognostic effect based on patient age. Specifically, among younger patients CA 19-9 serum level, vascular invasion, poor tumor grade, periductal invasion, and adjuvant radiotherapy were independent predictors of OS, while only periductal invasion and tumor grade were predictive of a worse OS among the elderly. Similarly, periductal invasion and nodal disease were associated with poor DFS among younger patients, while only poor tumor differentiation was associated with a worse DFS among the elderly. Consistent with the findings of the current study, Mavros et al. in a systematic review identified poor tumor differentiation, nodal disease, and vascular invasion as most predictive of survival among patients undergoing surgery for ICC [3]. In a separate study, Menon and colleagues reported that, while age was not independently associated with worse OS or DFS, other disease characteristics such as the number of tumors as well preexisting comorbidities were predictive of survival [1]. Taken together these findings suggest that hepatic surgery should not be denied to elderly patients. While the risk of perioperative morbidity may be higher in the elderly, elderly patients can enjoy comparable long-term outcomes compared with non-elderly patients. Rather, the collective data suggest that tumor specific factors, and not age itself determines prognosis.

The current study has several limitations. Given the retrospective nature of the study, no data on performance status was available. Although the ASA score was used as a surrogate for performance status/ comorbidity, it is possible that potential confounding due to case mix may have been unaccounted for. In particular the lack of data on comorbidities is a limitation of the study, although the ASA score was available in the majority of patients. Additionally, as data were collected from multiple institutions, the pre-, intra-, and postoperative management across participating institutions may have varied. However, the use of data collected from multiple institutions allowed for a larger sample size and greater generalizability of the results. Despite this, the sample size for the matched cohorts was relatively small $(n=129)$, which in turn limited the statistical power of some analyses. Lastly, despite the use of a propensity score matching algorithm, it is possible that there was some selection bias in the patient groups that remained unaccounted for due to the retrospective nature of the database.

In conclusion, elderly patients undergoing liver surgery for ICC demonstrated an increased risk of perioperative complications, but comparable long-term DFS and OS compared with younger patients. Rather, tumor characteristics, such as lymph node metastasis and periductal invasion, were more predictive of worse DFS. As such, age alone should not be a contraindication for hepatic resection and surgery should be considered in all cases, where disease is amenable to extirpation. In the future, more robust tools will be necessary to identify those elderly patients at greatest risk for poor postoperative outcomes following hepatic surgery for ICC.

\section{REFERENCES}

1. El-Serag HB, Engels EA, Landgren O, et al.: Risk of hepatobiliary and pancreatic cancers after hepatitis $\mathrm{C}$ virus infection: $\mathrm{A}$ population-based study of U.S. veterans. Hepatology 2009;49: $116-123$

2. Endo I, Gonen M, Yopp AC, et al.: Intrahepatic cholangiocarcinoma: Rising frequency, improved survival, and determinants of outcome after resection. Ann Surg 2008;248:84-96.

3. Malhi H, Gores GJ: Cholangiocarcinoma: Modern advances in understanding a deadly old disease. J Hepatol 2006;45:856-867.

4. Shaib YH, Davila JA, McGlynn K, et al.: Rising incidence of intrahepatic cholangiocarcinoma in the United States: A true increase? J Hepatol 2004;40:472-477.

5. Welzel TM, McGlynn KA, Hsing AW, et al.: Impact of classification of hilar cholangiocarcinomas (Klatskin tumors) on the incidence of intra- and extrahepatic cholangiocarcinoma in the United States. J Natl Cancer Inst 2006;98:873-875.

6. Khan SA, Toledano MB, Taylor-Robinson SD: Epidemiology, risk factors, and pathogenesis of cholangiocarcinoma. HPB 2008;10: $77-82$.

7. Altekruse SF, Petrick JL, Rolin AI, et al.: Geographic variation of intrahepatic cholangiocarcinoma, extrahepatic cholangiocarcinoma, and hepatocellular carcinoma in the United States. PLoS ONE 2015;10:e0120574.

8. de Jong MC, Nathan H, Sotiropoulos GC, et al.: Intrahepatic cholangiocarcinoma: An international multi-institutional analysis of prognostic factors and lymph node assessment. J Clin Oncol 2011;29:3140-3145.

9. Hyder O, Marques H, Pulitano C, et al.: A Nomogram to Predict Long-term Survival After Resection for Intrahepatic Cholangiocarcinoma: An Eastern and Western Experience. JAMA Surg 2014;149:432-438.

10. Spolverato G, Yakoob MY, Kim Y, et al.: The Impact of surgical margin status on long-term outcome after resection for intrahepatic cholangiocarcinoma. Ann Surg Oncol 2015;22:4020-4028.

11. Spolverato G, Ejaz A, Kim Y, et al.: Tumor size predicts vascular invasion and histologic grade among patients undergoing resection of intrahepatic cholangiocarcinoma. J Gastrointest Surg 2014;18: 1284-1291.

12. Spolverato G, Kim Y, Ejaz A, et al.: Conditional probability of long-term survival after liver resection for intrahepatic cholangiocarcinoma: A multi-institutional analysis of 535 patients. JAMA Surg 2015;150:538-545.

13. Spolverato G, Kim Y, Alexandrescu S, et al.: Is hepatic resection for large or multifocal intrahepatic cholangiocarcinoma justified? 
Results from a multi-institutional collaboration. Ann Surg Oncol 2015;22:2218-2225.

14. de la Fuente SG, Bennett KM, Scarborough JE: Functional status determines postoperative outcomes in elderly patients undergoing hepatic resections. J Surg Oncol 2013;107:865-870.

15. Tsukioka G, Kakizaki S, Sohara N, et al.: Hepatocellular carcinoma in extremely elderly patients: An analysis of clinical characteristics, prognosis and patient survival. World J Gastroenterol 2006;12: 48-53.

16. Kaibori M, Matsui K, Ishizaki M, et al.: Hepatic resection for hepatocellular carcinoma in the elderly. J Surg Oncol 2009;99: 154-160.

17. Adam R, Frilling A, Elias D, et al.: Liver resection of colorectal metastases in elderly patients. Br J Surg 2010;97:366-376.

18. Mirici-Cappa F, Gramenzi A, Santi V, et al.: Treatments for hepatocellular carcinoma in elderly patients are as effective as in younger patients: A 20-year multicentre experience. Gut 2010;59: 387-396.

19. Siegel AB, Lim EA, Wang S, et al.: Diabetes, body mass index, and outcomes in hepatocellular carcinoma patients undergoing liver transplantation. Transplantation 2012;94:539-543.

20. Takahashi H, Mizuta T, Kawazoe S, et al.: Efficacy and safety of radiofrequency ablation for elderly hepatocellular carcinoma patients. Hepatol Res 2010;40:997-1005.
21. Edge S, Byrd DR, Compton CC, et al.: AJCC Cancer Staging Manual, 7th edn. New York, NY: Springer; 2010.

22. Couinaud C: Liver anatomy: Portal (and suprahepatic) or biliary segmentation. Dig Surg 1999;16:459-467.

23. Dindo D, Demartines N, Clavien PA: Classification of surgical complications: A new proposal with evaluation in a cohort of 6336 patients and results of a survey. Ann Surg 2004;240:205213.

24. Siegel RL, Miller KD, Jemal A: Cancer statistics, 2015. CA Cancer J Clin 2015;65:5-29.

25. Phan $\mathrm{K}, \mathrm{An} \mathrm{VV}, \mathrm{Ha} \mathrm{H}$, et al.: Hepatic resection for malignant liver tumours in the elderly: A systematic review and meta-analysis. ANZ J Surg 2015;85:815-822.

26. Delva F, Marien E, Fonck M, et al.: Factors influencing genera practitioners in the referral of elderly cancer patients. BMC Cancer 2011;11:5.

27. Protiere C, Viens P, Rousseau F, et al.: Prescribers' attitudes toward elderly breast cancer patients. Discrimination or empathy?. Crit Rev Oncol Hematol 2010;75:138-150.

28. NIH consensus conference. Adjuvant therapy for patients with colon and rectal cancer. JAMA 1990;264:1444-50.

29. Guadagnoli E, Shapiro C, Gurwitz JH, et al.: Age-related patterns of care: Evidence against ageism in the treatment of early-stage breast cancer. J Clin Oncol 1997;15:2338-2344. 